Supporting Information

\title{
Scaleup of a Single-Mode Microwave Reactor
}

Himanshu Goyal ${ }^{1}$, Ali Mehdad ${ }^{1,2}$, Raul F. Lobo ${ }^{1,2}$, Georgios D. Stefanidis ${ }^{3}$, Dionisios G. Vlachos $^{1,2 *}$

1. Delaware Energy Institute, University of Delaware, 221 Academy Street, Newark, Delaware 19716, United States

2. Department of Chemical and Biomolecular Engineering, University of Delaware, 150 Academy Street, Newark, Delaware 19716, United States

3. Department of Chemical Engineering, KU Leuven, Celestijnenlaan 200F, B-3001 Leuven, Belgium

*Corresponding author: vlachos@udel.edu 
Experimental temperature profiles for water, ethanol, and hexane used for developing the calibration curve.
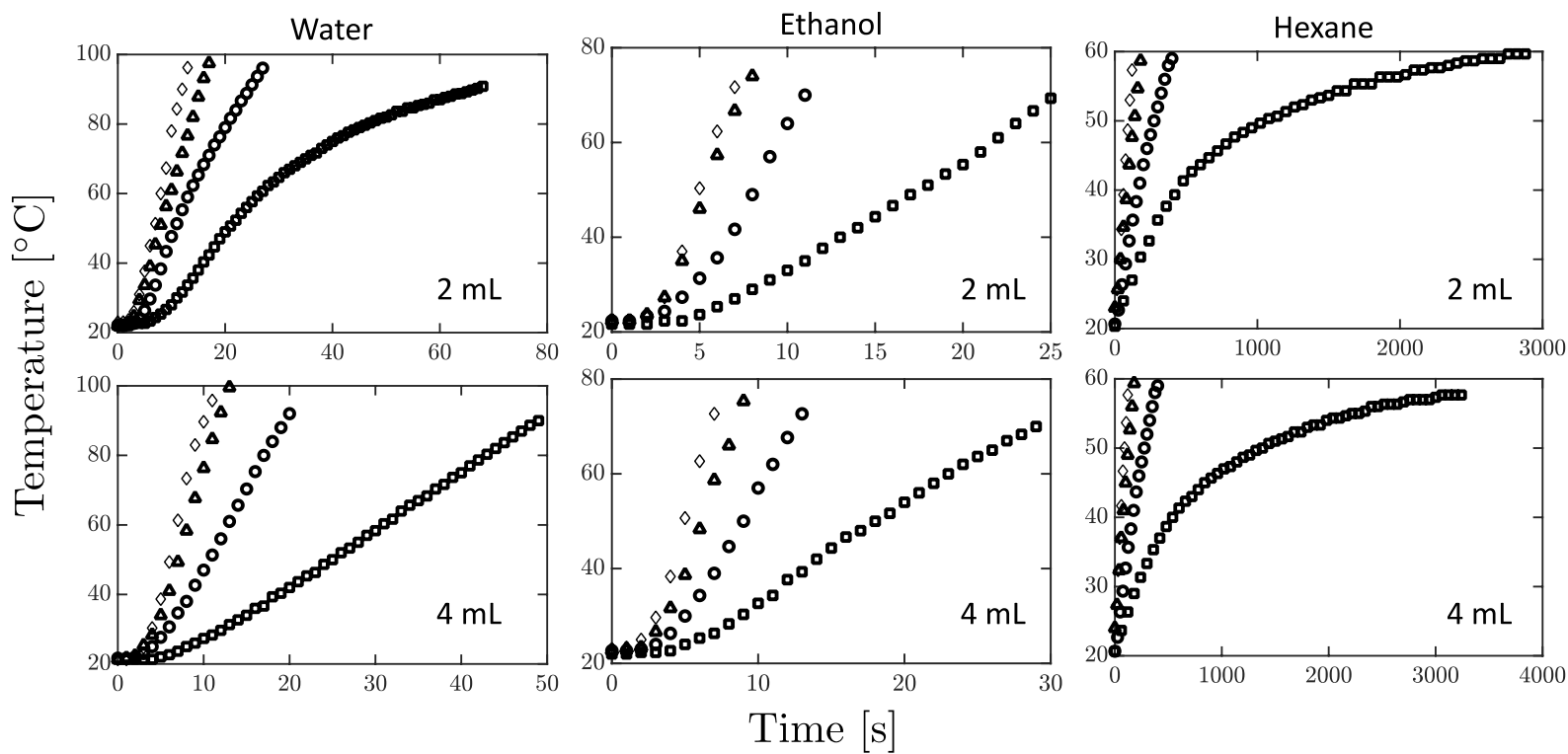

Figure S1: Experimental measurements of the temperature using a fiber optic probe during microwave heating of water, ethanol, and hexane. Input microwave powers used are $30 \mathrm{~W}$ (squares), $100 \mathrm{~W}$ (circles), $200 \mathrm{~W}$ (triangles), and $300 \mathrm{~W}$ (diamonds). 
Response surfaces for the average heating rates $\mathcal{J}_{i}^{\text {sim }}$ as a function of $f_{1}$ and $f_{2}$.
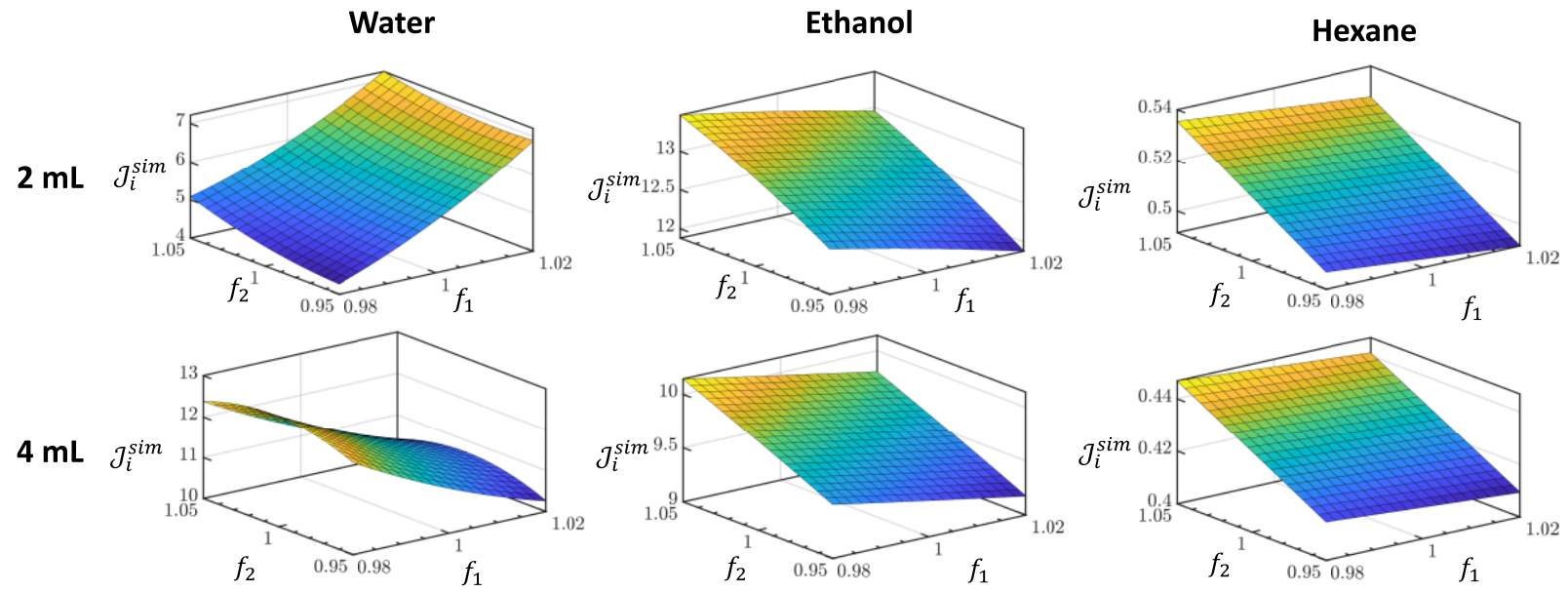

Figure S2: Response surfaces in the form of $2^{\text {nd }}$-order polynomial for the average heating rates $\mathcal{J}_{i}^{p}$ as a function of $f_{1}$ and $f_{2}$ at a microwave power of $300 \mathrm{~W}$. The coefficients are calculated using regression, in which the values of $f_{1}$ and $f_{2}$ are determined using the factorial design. 


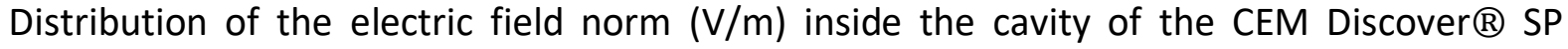
microwave reactor used in this work.

\section{(a) Empty cavity}

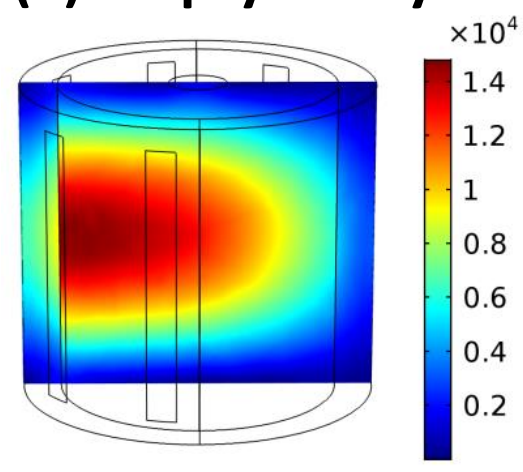

(c) Ethanol

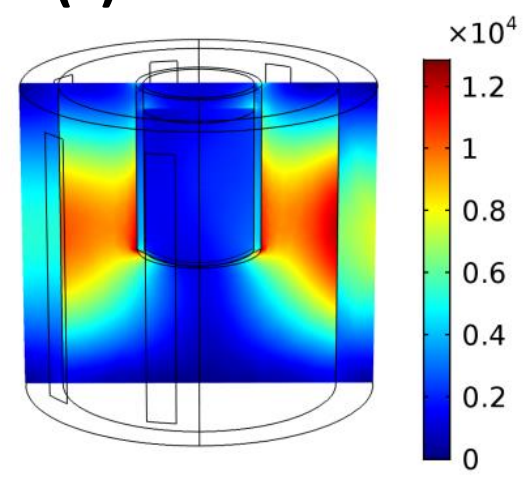

(b) Hexane

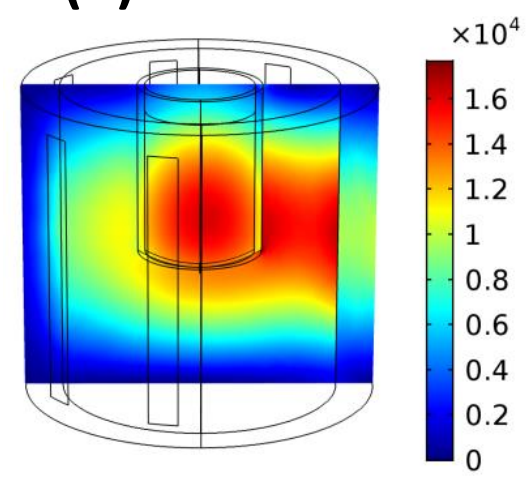

(d) Water

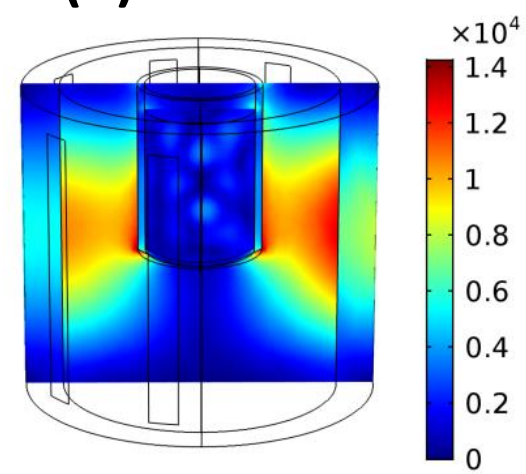

Figure S3: Electric field norm $(\mathrm{V} / \mathrm{m})$ inside the CEM Discover SP microwave reactor predicted using the COMSOL model with an input microwave power of $100 \mathrm{~W}$. (a) empty cavity; with a Pyrex vial of $30 \mathrm{~mm}$ inner diameter filled with (b) hexane; (c) ethanol; (d) water to a height of 40 $\mathrm{mm}$. 